\title{
Inovações tecnológicas e seus impactos na gestão de faturamento
}

\author{
José Felicio Frezza, Marta Rettelbusch de Bastos
}

\begin{abstract}
Resumo - As inovações tecnológicas oriundas do uso do Protocolo Internet (IP) estão cada vez mais permeando as redes de Telecomunicações, criando as chamadas Redes de Nova Geração (NGN). A convergência de redes e serviços está modificando as cadeias de valor dos setores de mídia e telecomunicações. As Empresas Operadoras de Telecomunicações Fixas e Móveis, assim como outras entidades prestadoras de serviços de valor adicionado, estão se associando, visando ao oferecimento de serviços convergentes aos usuários. Neste trabalho é apresentado e analisado um modelo de faturamento que evidencia os relacionamentos entre novas entidades que estão participando da cadeia de valor de novos serviços de telecomunicações.
\end{abstract}

Palavras-chave - Faturamento, Telecomunicações, Convergência.

Abstract - The technology innovations in consequence of the use of the Internet Protocol (IP) are more and more permeating all the Telecommunications networks, creating the New Generation Network (NGN). The services and networks convergence are modifying the value chain in media and telecommunications sector. The wired and mobile telecommunications companies are associating with other value-added service providers to offer converged services to their customers. This paper considers one billing model to show the relationship among some new entities that take part in the value chain of new telecom value-added services.

Keywords - Billing, Telecommunications, Convergence.

\section{INTRODUÇÃO}

Atualmente observa-se que está ocorrendo o que os provedores de soluções de telecom já preconizavam, que é a convergência das redes e serviços, ou seja, um mesmo serviço pode ser prestado por diversas redes e acessado através de diversos meios.

Segundo a Architectures for convergence, Business Communication Review, "A convergência é uma única estrutura, uma única arquitetura preparada para suportar todas as formas e canais de informação, em todas as formas de tecnologia de rede".

Hoje existem grupos de padronização tais como o International Telecommunication Union - Telecom (ITU-
T) [7], o TeleManagement Forum e o 3rd Generation Partnership Project (3GPP), dentre outros, que estão trabalhando na especificação de padrões, tais como: Internet Protocol Multimedia Subsystem (IMS). Esses padrões exercem forte poder direcionador da tecnologia. Com isto, podem-se criar futuros cenários de prestação de serviços com completa integração entre as redes de comunicações: fixas, móveis, satélite, TV a cabo e TV abertas, incluindo a TV digital, bem como as redes: Local Area Network (LAN), Wide Area Network (WAN) e Metropolitan Area Network (MAN). Inclui-se também a rede denominada Power Line Communication (PLC), que utiliza a fiação da rede elétrica como suporte.

Os paradigmas que vinham sendo utilizados em telecomunicações estão se mostrando inadequados para prever toda esta complexa mudança nos modelos de negócio decorrentes das inovações tecnológicas em telecomunicações. Em particular, os modelos de faturamento estão sofrendo grandes impactos para adequação aos novos negócios de telecomunicações.

Sob o ponto de vista do usuário, um dos principais meios de relacionamento com as empresas prestadoras de serviços é a fatura. Isto torna os sistemas de faturamento e compartilhamento de receitas de grande importância no apoio ao negócio.

A tecnologia atual permite o oferecimento de uma nova categoria de serviços com valor adicionado para o usuário, ou seja, serviços de conteúdo, ou de valor adicionado.

Para o provimento desses serviços, houve a necessidade d participação de novas entidades na cadeia de valor de produtos e serviços, visto que as operadoras de telecomunicações não possuem perfil para oferecer toda a gama de serviços que as novas tecnologias permitem. Isto gera novos modelos operacionais e de negócio.

São analisados neste trabalho, alguns impactos das inovações tecnológicas que estão proporcionando a convergência de redes e serviços, fazendo com que qualquer serviço possa ser provido por qualquer rede. Isto muda as cadeias de valores tradicionais dos setores de mídia e telecomunicações, tornando-as convergentes.

É também apresentado um modelo de sistemas de faturamento com os principais módulos e relacionamentos e outro modelo de faturamento incluindo provedores de serviços de valor adicionado e patrocinadores. 


\section{CONVERGÊNCIA DE REDES E SERVIÇOS}

Será abordada neste tópico a convergência das redes e dos serviços nos meios de acesso com e sem fio.

A Figura 1 a seguir ilustra a convergência nos meios de transmissão da informação e a independência que está ocorrendo entre os serviços e as redes de telecomunicações, embora os meios de acesso sejam específicos de cada rede, os serviços podem ser acessados por vários meios em diversas redes.

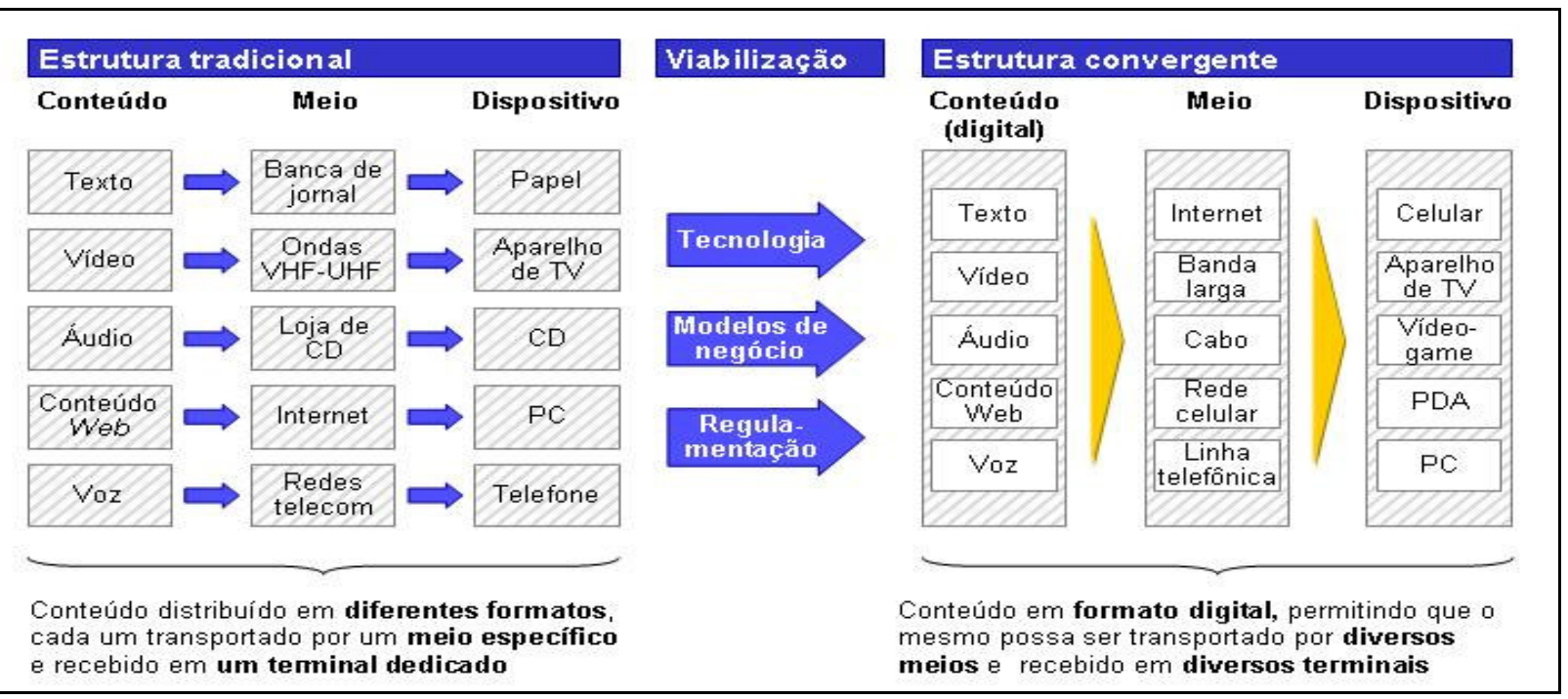

Fig. 1. Convergência na Transmissão de Informações [3]

\section{A. Convergência de Redes e Serviços com Fio}

Os serviços de acesso tradicionalmente oferecidos pelas operadoras de Serviços de Telefonia Fixo Comutado (STFC) eram analógicos, tais como o transporte de voz utilizando os aparelhos fixos residenciais e comerciais, bem como as linhas alugadas.

A digitalização da rede foi a primeira grande transformação que possibilitou o oferecimento de serviços tais como o de linhas de assinante digital assíncrona (Asymmetric Digital Subscriber Line - ADSL).

Uma segunda grande transformação pela qual as redes de telecomunicações estão passando atualmente é a utilização do Internet Protocol (IP). Os serviços de transporte de voz passaram a ser tratado como dados. A voz digitalizada passou a ser apenas um tipo de dado.

O computador, hoje, também é um terminal de acesso que, estando conectado à Internet com ou sem fio, permite a utilização de serviços de voz e dados. Existem aplicativos que permitem, além da navegação na Internet, a conversação utilizando Voz sobre IP (VoIP), download e upload de dados, vídeo conferência, video streaming mostrando imagens em tempo real de algum local do mundo etc.

Empresas provedoras de serviços de voz utilizando VoIP, utilizando-se de equipamentos denominados gateway,s, permitem ligações telefônicas entre computadores utilizando um software denominado softphone e aparelhos telefônicos tradicionais, ou seja, uma integração entre o mundo computacional e o das telecomunicações.

Organizações que possuem redes privadas de dados estão implantando VoIP para comunicação interna entre os seus ramais telefônicos, pois VoIP independe da distância entre os ramais e o custo fica com a rede de dados que, sendo privada, pode ter custo zero. As organizações estão implantando também gateways para comunicação com a rede de telefonia pública.

As redes privadas de TV a cabo e satélite estão também oferecendo serviços tradicionais de telecomunicações. Através de redes de TV é possível conectar-se à Internet e dispor de serviços de navegação, correio eletrônico, assim como telefonia VoIP.

As redes que estão sendo construídas com base nas tecnologias que utilizam os protocolos IP estão sendo denominadas de Redes de Nova Geração (NGN). Nestas redes, as centrais de comutação estão sendo substituídas por equipamentos denominados softswitch, responsáveis por sessões de comunicação entre vários tipos de meios de acesso.

As novas tecnologias estão propiciando uma convergência de redes, ou seja, os serviços estão se tornando independentes das redes e dos meios de acesso.

\section{B. Convergência de Redes e Serviços sem Fio}

As operadoras do Serviço Móvel Pessoal (SMP), mais notadamente do que as operadoras do STFC, também passaram a oferecer novos serviços com a digitalização da rede e a evolução dos terminais móveis (handset).

Atualmente está em franca ascensão o uso de serviços de acesso móvel. Dentre os mais difundidos estão os serviços de mensagens de texto através de Short Message System (SMS) e serviços Wireless Application Protocol (WAP), que permitem o acesso à Internet por meio dos terminais celulares (handset). Nas gerações 2,5 G e 3G, 
estão os serviços Multimídia Message System (MMS) que permitem a captura e visualização de imagens e filmes, serviços Smartphones que são "programas de software" que funcionam no celular, mesmo que este não esteja conectado da rede, ou seja, dão ao terminal características de computador. Há, também, os serviços baseados em localização Location Based System (LBS), que conseguem identificar precisamente a posição geográfica de um celular, utilizando-se de mecanismos de triangulação de sinal captado pelas antenas dos sistemas móveis ou por sinais de satélite conhecidos por GPS.

Para exemplificar, um serviço de valor adicionado que pode ser oferecido conjuntamente com os serviços LBS é o de monitoração se o celular está sempre dentro de uma região delimitada, e envio uma mensagem, quando ele sai da região. Este serviço poderia ser utilizado, por questão de segurança, entre pais e filhos.

Outro meio de acesso sem fio que está crescendo bastante é o Wireless Local Area Network (WLAN) ou Wireless Fidelity (Wi-Fi), que consiste em equipamentos de comunicação geralmente instalados em computadores portáteis, para estes acessarem a Internet sem fio. Existem também Personal Digital Assistant (PDA) e celulares com este tipo de acesso. Isto permite que esses equipamentos acessem a Internet diretamente, bastando que estejam em algum local que disponha de ponto de acesso Wi-Fi (hotspot), tal como um aeroporto ou hotel.

Outra rede analógica que está em processo de transformação para digital é a TV aberta. Esta rede é polêmica porque mais de $90 \%$ das pessoas do país tem acesso a ela. Uma premissa deste serviço é a gratuidade para o usuário final. Hoje a receita advém de patrocínios e propagandas.

\section{Cadeia de Valor Convergente}

O setor de mídia possui uma cadeia de valor que tradicionalmente produz conteúdo, empacota e distribui, não tendo relacionamento direto com o usuário. Por outro lado, a cadeia de valor tradicional de Telecomunicações não produz conteúdo. Entretanto, ela realiza a operação das redes e oferece serviços de acesso, possuindo relacionamento direto com os usuários dos serviços. $\mathrm{O}$ processo de convergência está criando uma nova cadeia de valor convergente, que está unindo desde a produção do conteúdo até o usuário deste conteúdo. A Figura 2 apresenta esta cadeia.

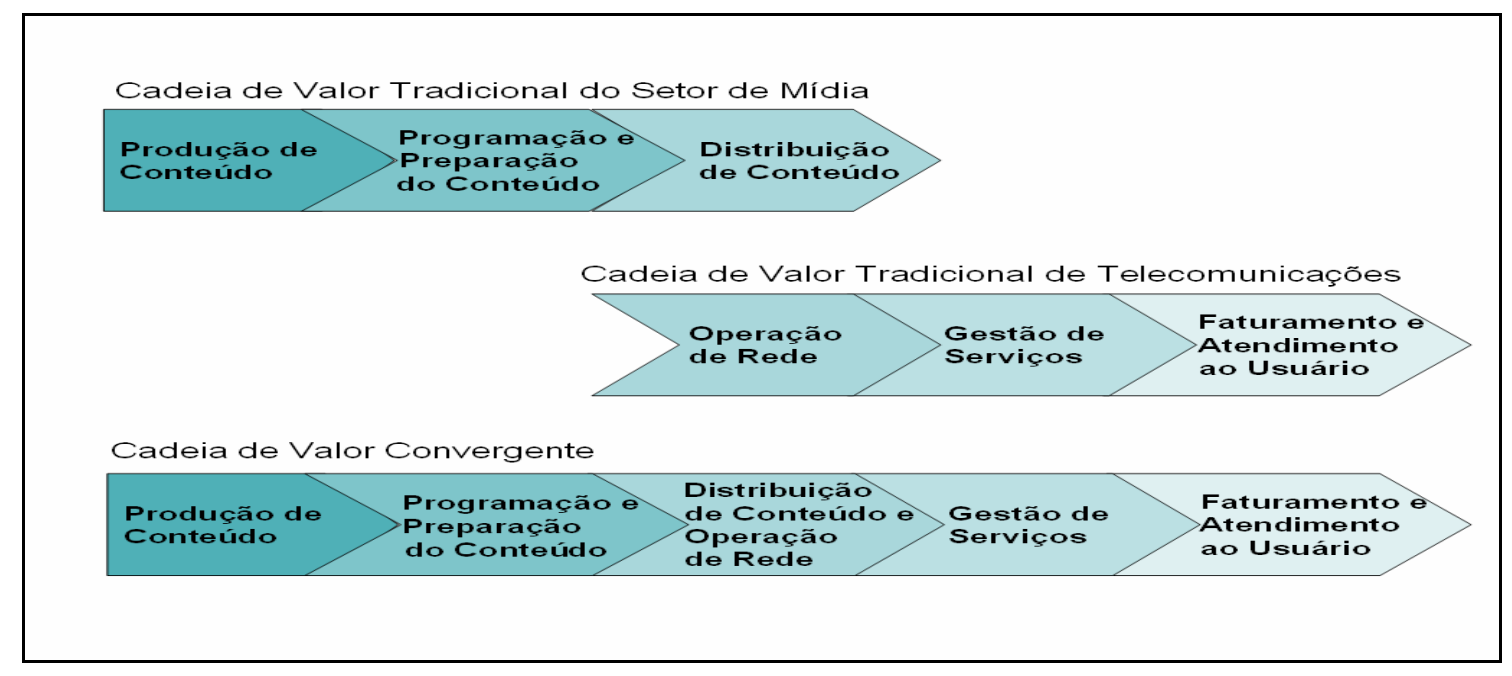

Fig. 2. Cadeia de valor convergente [4]

Novos negócios e oportunidades surgirão com a TV digital pública ou privada, propiciadas pela convergência de serviços. Podem ser citados serviços relacionados com interatividade, mobilidade e portabilidade, tais como: novos serviços SMS, comércio via TV (T-commerce), serviços bancários pela TV (T-banking), jogos interativos pela TV, dentre outros. Isto implicará em novos modelos de negócios, com novos atores e cadeias de valores.

A Inclusão Digital é outro aspecto importante que a TV digital, principalmente a pública, pode proporcionar com serviços de acesso a Internet e ensino à distância.

A convergência de serviços está sendo tratada em quatro aspectos: voz, dados, vídeo e mobilidade, sendo que os serviços que disponibilizam estas quatro características são denominados de Quadruple-Play.
Um dos fóruns de padronização que mais tem trabalhado em recomendações visando à convergência das diversas redes é o 3GPP [1]. As recomendações indicam a tecnologia IMS [2] como evolução das redes. O 3GPP está definindo as interfaces entre diversos módulos que compõem a tecnologia, sem entrar no mérito de como cada provedor de soluções desenvolve cada módulo, garantindo, assim, o inter funcionamento de módulos de provedores diferentes.

A seguir será apresentado um modelo em camadas, que possibilita uma visão de alguns sistemas e tecnologias envolvidos na convergência. 


\section{Modelo de Convergência em Camadas}

Este tópico apresenta um modelo em camadas nos níveis de rede, transporte, sessão, aplicação e de negócios. Mostra que os sistemas de suporte ao negócio (BSS) em especial os sistemas de faturamento (Billing) pré e póspagos necessitam tratar uma série de aplicações e serviços que cada vez mais independem das redes e mais que isto, podem utilizar várias redes para a prestação dos serviços.

A camada de transporte com a utilização comum de protocolos IP é que está possibilitando a independência das redes e o controle das sessões de prestação dos serviços está sendo feito por protocolos que possibilitam esta independência.

A Figura 3 ilustra um modelo em camadas, que mostra várias redes de acesso, onde o protocolo IP possibilita a convergência da prestação dos serviços de transporte e as sessões de comunicação são controladas por diversos protocolos. A camada de aplicação contém apenas alguns exemplos, que podem ser desde softphones para serviços de voz em redes com VoIP até sistemas de tarifação online de serviços pré-pagos que fornecem informações para os sistemas de faturamento (billing) gerarem os demonstrativos para serviços pré-pagos ou faturas para serviços pós-pagos, assim como realizarem toda a distribuição e controle de receitas a todas as entidades participantes na prestação dos serviços.

\begin{tabular}{|c|c|c|c|c|c|c|c|}
\hline Negócio & \multicolumn{2}{|c|}{$\begin{array}{l}\text { Billing } \\
\text { Pós-Pago }\end{array}$} & \multicolumn{2}{|c|}{$\begin{array}{l}\text { Billing } \\
\text { Pré-pago }\end{array}$} & \multicolumn{2}{|c|}{ BSS / ISS } & \\
\hline Aplicações & Voz & & ação & LB & & Jogos & ڤె. \\
\hline $\begin{array}{l}\text { Controle } \\
\text { de } \\
\text { Sessão } \\
\end{array}$ & \multicolumn{2}{|c|}{ SIP } & \multicolumn{2}{|c|}{$\begin{array}{l}\text { RADIUS } \\
\text { Diameter }\end{array}$} & \multicolumn{2}{|c|}{ SDAP } & @્ळ \\
\hline Transporte & \multicolumn{6}{|c|}{ Rede IP } & \\
\hline $\begin{array}{l}\text { Redes de } \\
\text { Acesso }\end{array}$ & xDSL & $\begin{array}{l}\text { TV } \\
\text { Cak }\end{array}$ & Saté & & $\mathrm{i}-\mathrm{Fi}$ & Celular & \\
\hline
\end{tabular}

Fig. 3. Modelo de Convergência em Camadas

Este modelo possibilita uma melhor compreensão dos serviços que poderão ser prestados num futuro próximo, através da utilização de tecnologias como o IMS.

Podem ser citados como futuros serviços [5], a utilização de um gerenciador de perfil de comunicações pessoais, ou seja, o usuário cadastra suas características em uma base de dados e pode especificar em qual meio de acesso deseja receber suas ligações telefônicas. Através do gerenciados o usuário pode dispor de apenas um número telefônico e programar para receber chamadas pela manhã em seu computador utilizando um softphone, na hora do almoço as ligações são encaminhadas para seu celular, a tarde são desviadas para uma caixa postal (voice mail) e a noite as ligações são completadas em seu terminal fixo, com aviso de quem está chamando na tela da TV.
A seguir, serão abordados alguns modelos de faturamento mostrando os relacionamentos entre algumas destas entidades.

\section{MODELOS DE FATURAMENTO PARA ATENDER A CONVERGÊNCIA DE SERVIÇOS}

Os modelos de faturamento para atender à convergência de serviços e os serviços de valor adicionado precisam contemplar várias entidades que compõem a cadeia de valor do serviço, tais como: provedores do conteúdo em si, programação de conteúdo e provedores de aplicações que preparam e adaptam este conteúdo possibilitando seu oferecimento comercial.

Cada entidade participante da cadeia de valor precisa ter visão clara e transparente do volume de uso dos serviços para viabilizar o gerenciamento das receitas e despesas, de acordo com os contratos firmados com as demais entidades.

Atualmente existem vários organismos internacionais trabalhando na regulamentação e na padronização das interfaces e funcionalidades visando à integração de sistemas de diferentes fornecedores nestes novos modelos de faturamento.

\section{A. Processos de Faturamento}

A Global Billing Association (GBA) é uma organização sem fins lucrativos, focada em empresas Operadoras e provedores de serviços. A GBA possui um modelo que identifica os principais módulos que constituem um sistema de faturamento.

O TeleManagement Forum [8] é um consórcio internacional de fornecedores de sistemas e provedores de serviços no mercado de telecomunicações para estabelecer padrões de uso em diversos segmentos, incluindo especificações sobre a nova geração de serviços e sistemas de suporte Next Generation Operational Support Systems (NGOSS). Fornece também o Mapa de Aplicações em Telecomunicações (TAM), que mostra as principais aplicações de faturamento, nos níveis de Recursos, Serviços, Clientes e Fornecedores/Parceiros, e o modelo eTOM que analisa os processos de faturamento.

Baseado no modelo de faturamento da GBA, no Mapa de Aplicações TAM e no modelo eTOM, foi possível elaborar, segundo a visão dos autores, um modelo mais detalhado, mostrando os principais módulos de um Sistema de Faturamento e seus principais relacionamentos, com o objetivo de facilitar a compreensão das funcionalidades de cada módulo, conforme apresentado na Figura 4.

A Figura 4 apresenta, na horizontal, as quatro Gerências de Operações do modelo eTOM, onde estão mostrados os principais processos eTOM relacionados ao faturamento. Alguns processos estão mais detalhados do que na recomendação. Os processos realçados são os que compõem efetivamente os sistemas de faturamento. Estão detalhados também alguns dos principais fluxos entre os processos. 


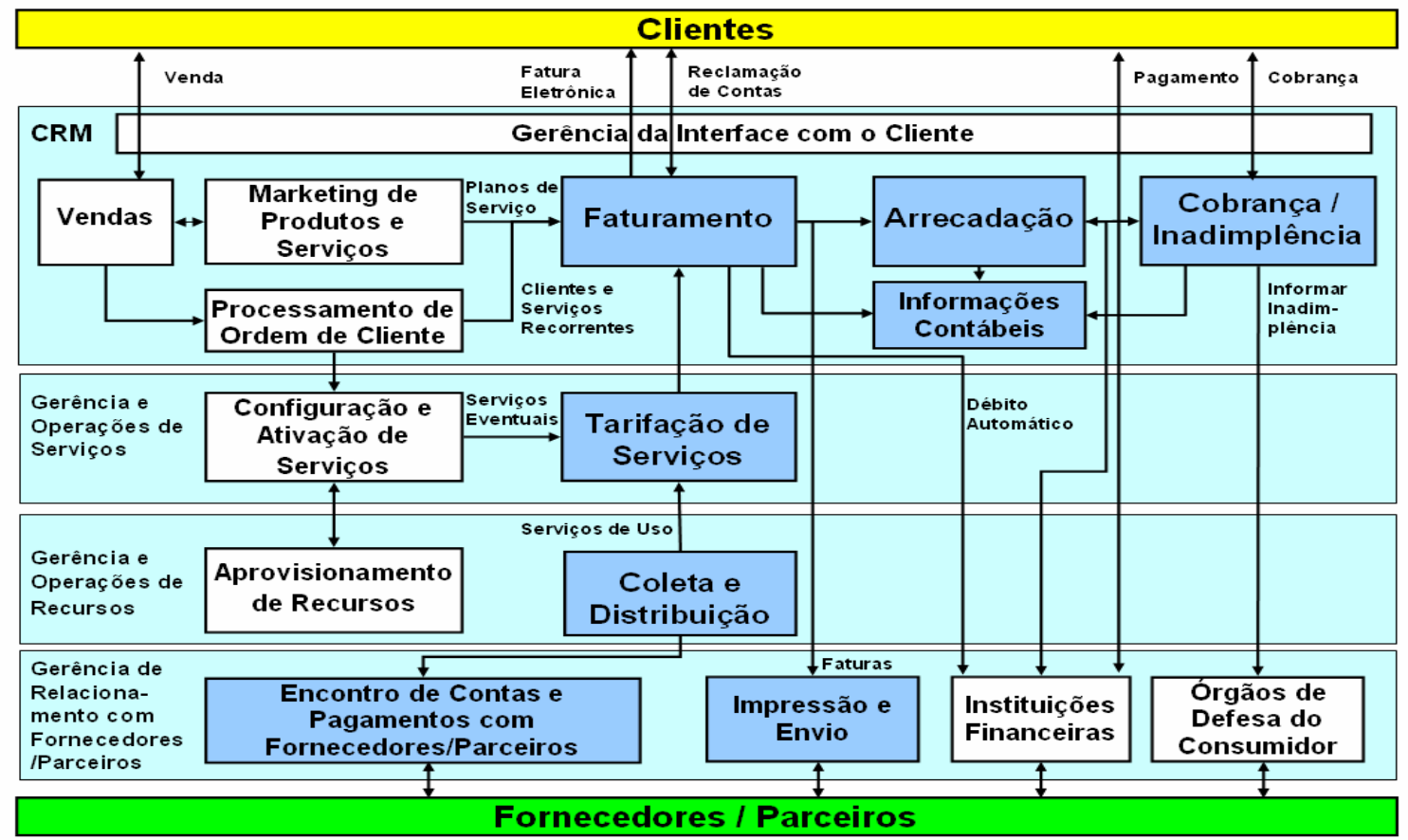

Fig 4. Sistemas de faturamento baseado no eTOM [4]

Os clientes são os usuários finais que adquirem os produtos e usam os serviços, realizando os respectivos pagamentos. É para a satisfação dos clientes que as empresas trabalham, pois as receitas das empresas de Telecomunicações vêm essencialmente dos clientes, de forma direta ou indireta. De forma direta, através do faturamento e arrecadação dos produtos e serviços. De forma indireta, pelo uso de seus recursos de rede por clientes de outras Prestadoras, neste caso recebendo as respectivas remunerações via contratos de interconexão e co-billing com outras empresas. Por outro lado, no caso de clientes de uma empresa utilizarem recursos de outra, esta também deve repassar os valores arrecadados via interconexão e co-billing.

\section{B. Modelo de Faturamento e Interconexão}

A organização mundial IPDR.org [6] é composta por representantes dos principais fornecedores de equipamentos e sistemas nesta área e possuem padrões para o registro de serviços IP e interfaces entre sistemas. Entidade onde os autores participaram no desenvolvimento de alguns modelos.

Este trabalho apresenta na Figura 5 um modelo de faturamento que representa uma forma de utilização de serviços de valor adicionado providos por provedores de serviços, operadoras e patrocinadores.

Os provedores de serviços são constituídos por provedores de aplicações e de serviços de conteúdo, sendo que estes podem ser entidades separadas ou uma única. Desta forma, o relacionamento da operadora pode ser com uma entidade única ou com ambas.

A operadora de telecomunicações provedora do meio de acesso ao usuário é quem realiza a Mediação dos serviços, ou seja, a coleta e o registro das informações de uso dos serviços. Esses dados são enviados para vários Sistemas de Suporte ao Negócio (BSS), destacando-se o sistema de Faturamento, que é o responsável pela geração das faturas e envio aos usuários, bem como pela arrecadação dos pagamentos efetuados. São consideradas as participações dos Patrocinadores, valorando cada serviço de acordo com os planos de negócio.

No caso de serviços pré-pagos, um sistema on-line debita do saldo de cada usuário o valor da utilização dos serviços. No entanto, o registro do uso tem que ser disponibilizado para as outras entidades participantes.

As operadoras são responsáveis pelo acerto dos valores devidos a cada entidade e possuem Sistemas de Interconexão (Settlement System) responsáveis por esse acerto. Esses sistemas realizam o faturamento, a arrecadação dos valores devidos pelos patrocinadores e o repasse dos valores devidos aos provedores, em função dos valores arrecadados dos usuários e dos patrocinadores.

Exemplificando, podemos ter um serviço onde o valor faturado é $70 \%$ pago pelo usuário (U) e $30 \%$ pago pelos patrocinadores $(\mathrm{P})$. Do total arrecadado, $20 \%$ é repassado ao provedor de conteúdo (C) e $30 \%$ ao provedor do serviço $(\mathrm{S})$, ficando $50 \%$ para a empresa prestadora de telecomunicações $(\mathrm{T})$. Portanto, $\mathrm{P}+\mathrm{U}=\mathrm{C}+\mathrm{S}+\mathrm{T}$.

Os provedores de serviços são as entidades que criam e mantêm os serviços. Utilizam-se dos meios da operadora para a disponibilização dos serviços aos usuários. Eles também monitoram e gerenciam a utilização dos serviços providos, para possibilitar o respectivo faturamento perante a operadora.

As entidades patrocinadoras também realizam o monitoramento e o acompanhamento do uso dos serviços, geralmente consultando as informações da operadora, pois 
necessitam desses dados para o acompanhamento de todo o processo e conferência dos valores patrocinados.

Este modelo inclui uma entidade que patrocina parte do valor dos serviços em função da propaganda feita ao usuário, ou seja, oferece a vantagem do usuário pagar menos por um serviço e para as empresas abre um novo e grande mercado publicitário.

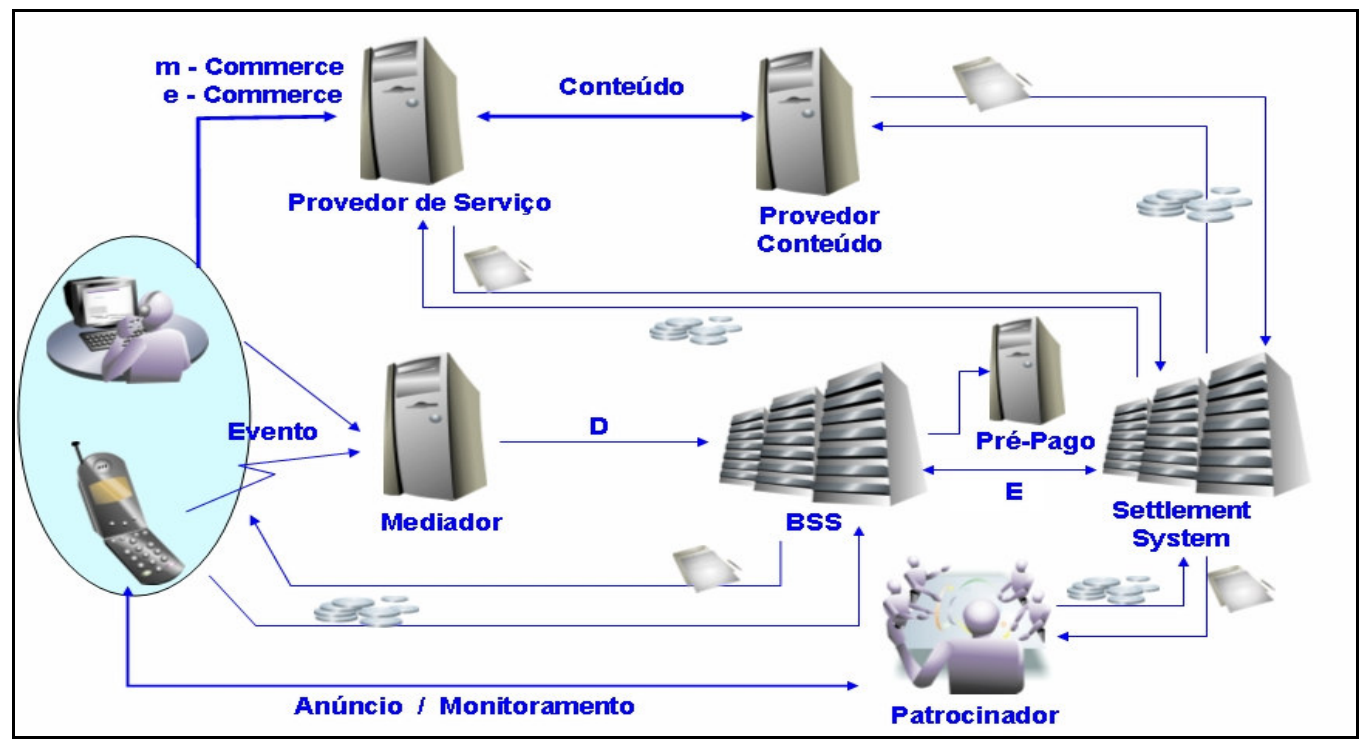

Fig 5. Modelo de Faturamento com Provedores de Serviços e Patrocinadores.

\section{CONCLUSÕES}

A digitalização das redes e dos meios de acesso, bem como o uso de IP, possibilitaram a convergência das redes e dos serviços, assim como o aumento da prestação de nova gama de serviços. Hoje se pode acessar os mesmos serviços através de um computador, via rede fixa, por um terminal móvel ou usando redes de TV fechadas.

Foram apresentados modelos de sistemas de faturamento, seus principais módulos e relacionamentos, provedores de serviços de valor adicionado e patrocinadores.

O faturamento de serviços convergentes e de conteúdo representa uma mudança de paradigma para os sistemas atuais de faturamento. Estão sendo requeridas funcionalidades tais como: análise de conteúdo, monitoramento de uso, remuneração e repasse de toda cadeia de valor envolvida na prestação dos serviços. Tais funcionalidades exigem flexibilidade dos sistemas atuais, bem como integração com outros sistemas, tanto de operadoras quanto das demais entidades participantes.

Este trabalho procurou mostrar alguns dos principais pontos de impacto na gestão de faturamento, em função de novos modelos de negócio que estão surgindo devido à convergência de redes e serviços.

\section{REFERÊNCIAS}

[1] 3GPP: 3rd Generation Partnership Project. TECHNICAL REPORT TR 22.115, v. 6.7.0, Service aspects; Charging and Billing (Release 7). 2006. Disponível em: <http://www.3gpp.org/>. Acesso em: 16 abr. 2007.

[2] 3GPP: 3rd Generation Partnership Project. TECHNICAL REPORT TR 32.240, v. 7.0.0, Charging architectures and principles (Release
7). 2006. Disponível em: <http://www.3gpp.org/>. Acesso em: 16 abr. 2007.

[3] ARAÚJO, M.C.P. Convergência digital. In: FUTURECOM, 2006, Florianópolis. Anais eletrônicos... Curitiba: Provisuale Participações, $2006 . \quad$ Disponível em: <http://www.futurecom2007.com.br/s_pal2006.html>. Acesso em: 14 mar. 2007.

[4] FREZZA, J.F. Modelos de Faturamento em Telecomunicações Decorrentes da Convergência das Redes e Serviços. mar. 2007. 149f. Dissertação (Mestrado em Engenharia). Pontifícia Universidade Católica de Campinas. Campinas, 2007. Disponível em: $<$ http://www.bibliotecadigital.puc-

campinas.edu.br/tde_busca/arquivo.php?codArquivo=201>. Acesso em: 25 abr. 2007.

[5] GORTON, D. Real-time interactive communications: beyond VoIP (Telcordia Technologies). In: TELEMANAGEMENT FORUM REGIONAL SUMMIT. Rio de Janeiro, 2005. Anais eletrônicos... Rio de Janeiro: TeleManagement Forum, 2005. Disponível em: $<$ http://www.tmforum.org/browse.aspx ?catid=2360>. Acesso em: 15 abr. 2007.

[6] IPDR.org, IPDR-SPS1.OID-R6 - Supplier/Partner Settlements for Next Generation IP-Based Services, 2003 Disponível em: <http://www.ipdr.org>. Acesso em: 15 abr. 2007.

[7] ITU-T, Series D. General tariff principles - Costs and Value of Services Rendered as Factors in the Fixing of Rates, D.5, 1988 a 2006. Disponível em: <http://www.itu-t.int/home>. Acesso em: 15 abr. 2007.

[8] TeleManagement Forum ${ }^{\mathrm{TM}}$, GB920 - New Generation Operational Support Systems ("NGOSS") - Architecture Overview, Public Version 1.5, November 2000. Disponível em: $<$ http://www.tmforum.org/>. Acesso em: 15 abr. 2007.

José Felício Frezza, Diretoria de Billing, Fundação CPqD, Campinas, Brasil, e-mail: frezza@cpqd.com.br.

Marta Rettelbusch de Bastos, Diretoria de Soluções de Inteligência de Negócios, Fundação CPqD, PUC-Campinas, Campinas, Brasil, e-mail: marta@cpqd.com.br.

Este trabalho é resultado de dissertação do curso de Mestrado Profissional em Gestão de Redes de Telecomunicações, em convênio entre a PUC-Campinas e a Fundação CPqD. 